\title{
Axes determination for segmented true-coaxial HPGe detectors
}

 \\ ${ }^{1}$ Max-Planck-Institut für Physik, München, Germany \\ ${ }^{2}$ Department of Physics, Durham University, Durham, UK
}

Received: 22 December 2011 / Revised: 24 January 2012 / Published online: 20 March 2012

(C) The Author(s) 2012. This article is published with open access at Springerlink.com

\begin{abstract}
A fast method to determine the crystallographic axes of segmented true-coaxial high-purity germanium detectors is presented. It is based on the analysis of segmentoccupancy patterns obtained by irradiation with radioactive sources. The measured patterns are compared to predictions for different axes orientations. The predictions require a simulation of the trajectories of the charge carriers taking the transverse anisotropy of their drift into account.
\end{abstract}

\section{Introduction}

High purity germanium detectors, HPGeDs, are used in a wide variety of applications in particle and nuclear physics [1-4]. Segmented detectors can be used to disentangle event topologies [5-7]. The detectors considered here are segmented cylindrical true-coaxial detectors. The segmentation in azimuth angle ${ }^{1} \phi$ is the decisive feature for the proposed method.

The orientation of the crystallographic axes of a germanium detector is important for many analyses where pulse shapes are used. The difference between the charge-carrier mobilities along the crystallographic axes $\langle 110\rangle$ and $\langle 100\rangle$ is significant. It is called longitudinal anisotropy. Experimental data on hole mobility exist for the $\langle 111\rangle$ and $\langle 100\rangle$ axes [8]. Relevant here is the plane containing the $\langle 110\rangle$ and $\langle 100\rangle$ axes. Appropriate calculations [9] imply that the difference of the velocities of the holes along the $\langle 110\rangle$ and $\langle 100\rangle$ axes is about $10 \%$ for realistic values of the electric field. The different charge carrier velocities result in different rise times of pulses originating at equal distances from the electrode. The differences in rise times between pulses along the $\langle 110\rangle$

\footnotetext{
${ }^{1} \mathrm{~A}$ cylindrical coordinate system is used with the origin at the center of the detector.

a e-mail: volynets@mppmu.mpg.de

${ }^{b}$ Now: Institute for the Physics and Mathematics of the Universe, Tokyo University, Japan
}

and $\langle 100\rangle$ is typically around $10 \%[9,10]$ depending on the relative location of the charge deposits to the crystal axes. In addition, the paths of charge carriers not drifting along the crystallographic axes are bent; this is called transverse anisotropy. The anisotropies have to be taken into account in so called pulse shape analyses $[11,12]$ and whenever simulated pulses [9] are compared to data.

The crystallographic $\langle 001\rangle$-axis of a cylindrical germanium detector is usually aligned with the $z$ axis. The position of the $\langle 110\rangle$ axis, $\phi_{\langle 110\rangle}$, is, however, a priori not known after the detector is processed. Therefore the orientation of the $\langle 110\rangle$ axis with respect to the segment boundaries has to be determined during the characterization of the detector.

This paper presents a comparison of two methods to determine the axes. The first method is based on scanning the detector. It is widely used, well proven and based on data only. However, it requires that the detector is mounted in a special test setup with a movable source and it is time consuming. The second method involves the comparison to Monte Carlo simulation. Its advantages are that it is fast and can be performed in any configuration, even if the detector is part of a complex detector system. The determination of the axes is required to have an accuracy of about $5^{\circ}$. A simple application using the orientation of the axes is the calculation of the probability that a neutrinoless double beta decay event actually produces a signal in two segments of a detector, even though it is a so called single-site event. This requires that the knowledge about the axes orientation is sufficient to calculate whether a drifting charge cloud gets separated across segment boundaries. The charge cloud representing a neutrinoless double beta decay event has a diameter of about $2 \mathrm{~mm}$ [13], and thus covers about $5^{\circ}$ at a radius of $25 \mathrm{~mm}$ in a cylindrical detector.

\section{Detector and test environment}

The detector used for this study was an n-type true-coaxial 18-fold segmented detector with a diameter of $75 \mathrm{~mm}$, a 
height of $70 \mathrm{~mm}$ and an inner bore of $10 \mathrm{~mm}$. It was produced by Canberra, France. The segmentation scheme is three in $z$ and six in $\phi$. The layout is depicted in Fig. 1. The first such detector was characterized in detail [10] including the determination of the orientation of the crystallographic axes. The second detector of the series, used for this study, was previously used to test the performance of such a device while submerged in a cryogenic liquid [14] and the temperature dependence of pulse lengths [15].

The density of electrically active impurities, $\rho_{\text {imp }}$, was given by the manufacturer as $0.35 \cdot 10^{10} \mathrm{~cm}^{-3}$ at the top and $0.55 \cdot 10^{10} \mathrm{~cm}^{-3}$ at the bottom of the detector. The change in impurities is assumed to be linear with height, $z$. The operational voltage was $2000 \mathrm{~V}$. At the time of the measurements, the detector drew a leakage current of up to $100 \mathrm{nA}$,

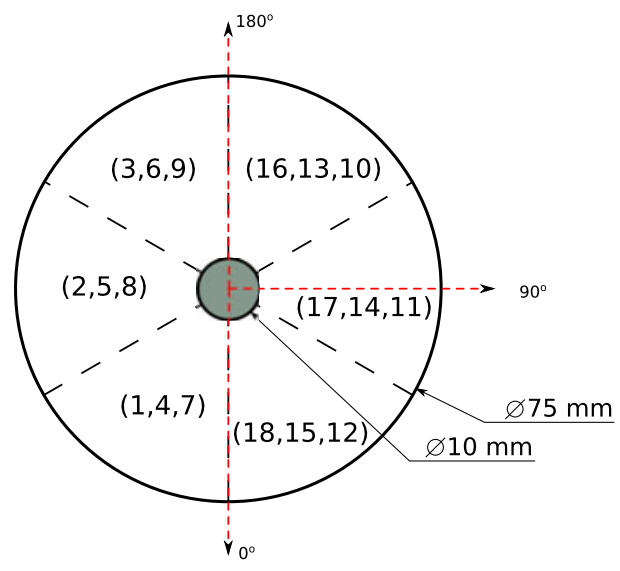

(a)

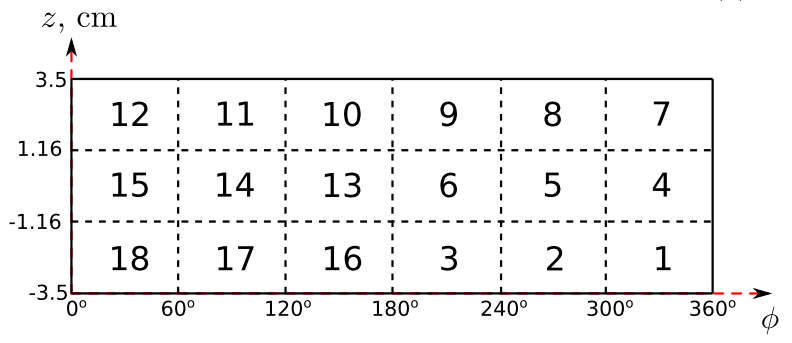

Fig. 1 (a) Coordinate system with the $x$ axis chosen to be on the 4-15 segment boundary. Segment boundaries are indicated as dashed lines, coordinate axes as dotted lines. Numbers in parenthesis are segment numbers as in (b). (b) Detector segment layout in $(\phi, z)$ coordinates all through segment 9 . It was, however, fully functional. The only effect was that the energy resolutions in segment 9 and the core had deteriorated.

For all measurements, the detector was mounted inside the vacuum cryostat $\mathrm{K} 1$ [15]. The detector was scanned in $\phi$ at $z=0$ with a ${ }^{152}$ Eu source placed at $r=9.6 \mathrm{~cm}$. It was also irradiated from the top $(z=18.5 \mathrm{~cm}, r=0 \mathrm{~cm})$ with a ${ }^{228} \mathrm{Th}$ and a ${ }^{60} \mathrm{Co}$ source. The ${ }^{228} \mathrm{Th}$ source was also placed at the side $\left(z=-2.3 \mathrm{~cm}, r=17.6 \mathrm{~cm}, \phi=155^{\circ}\right)$, in front of segment 16 .

\section{Detector scan}

A well established way to find the crystallographic axes is to perform an azimuthal scan, where a source with a low energy gamma line, like ${ }^{152} \mathrm{Eu}$ with its $122 \mathrm{keV}$ line, is moved around the detector in small steps in $\phi$. Low energy gamma rays predominantly deposit their energy close to the surface. The rise times of the resulting pulses vary with $\phi$, reaching a maximum for the drift along the $\langle 110\rangle$ axes.

A scan was performed with a collimated $40 \mathrm{kBq}{ }^{152} \mathrm{Eu}$ source with a $1 \sigma$ beam spot diameter of about $5 \mathrm{~mm}$ and a step size of $10^{\circ}$ in $\phi$. The events were selected using an energy window of $10 \mathrm{keV}$. The rise time of a given pulse was determined by fitting a simulated pulse to the measured one. This is described in detail elsewhere $[9,15]$. This method uses all the available information about a measured pulse while suppressing the influence of noise. For every $\phi$ position, the average rise time, $t_{r}$, was computed for all pulses, for which $\chi^{2} /$ ndof $<1.5$ for the fit with the simulated pulse. Figure 2 shows the dependence of $t_{r}$ on the angle of the source position, $\phi$. The data were fitted with the function

$t_{r}(\phi)=A+B \cdot \sin \left(\frac{2 \pi}{90}\left(\phi+\phi_{\langle 110\rangle}\right)\right)$,

where $A, B$ and $\phi_{\langle 110\rangle}$ are the free parameters. The coordinate system was chosen such that the 4-15 segment boundary was at $0^{\circ}$.

A study of the systematic uncertainties was performed. The energy window, in which events were selected, was changed to $5 \mathrm{keV}$ and $20 \mathrm{keV}$. In addition, the $\chi^{2}$ used for the
Fig. 2 Dependence of rise time on the azimuth angle $\phi$ of the source position. The points are the data with statistical uncertainties. If the uncertainty bars are not visible, they are smaller than the points. The line represents the fit described in the text. The vertical lines indicate the segment boundaries; the numbers are segment numbers

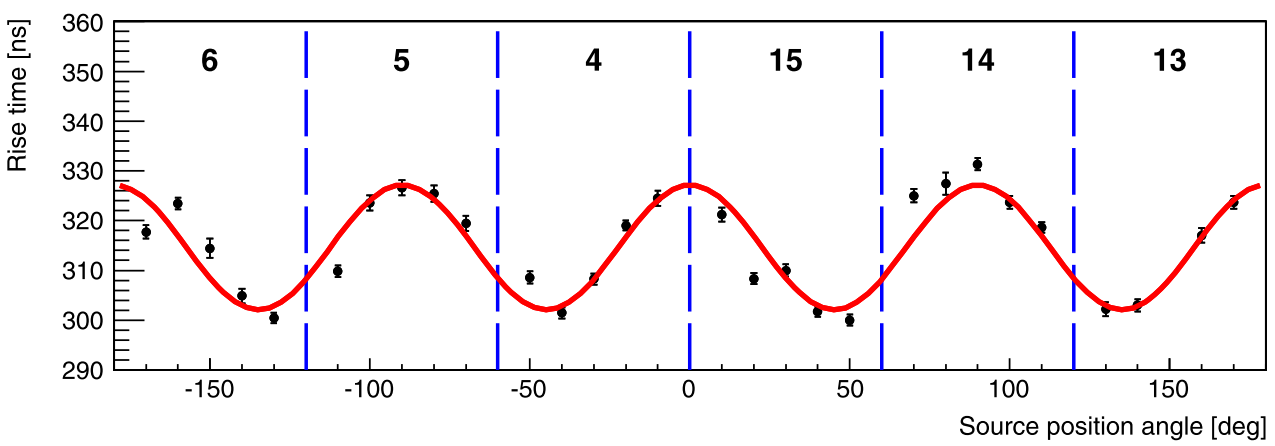


pulse selection was modified from 1.5 to 1.2 and 1.8. In both cases, a systematic uncertainty of $0.5^{\circ}$ was deduced. The dominating systematic uncertainty is connected to the placement of the detector within the cryostat. This could only be controlled to $3^{\circ}$. The total systematic uncertainty was determined by adding the individual contributions in quadrature. The statistical uncertainty, as determined by the fit, is $0.4^{\circ}$. The final result is:

$\phi_{\langle 110\rangle}=-0.2^{\circ} \pm 0.4^{\circ}$ (stat.) $\pm 3.1^{\circ}$ (syst).

\section{Occupancy method}

The drift of the charge carriers within a germanium detector only follows radial lines along the crystallographic axes. The transverse anisotropy causes bent trajectories elsewhere in the crystal [9, Fig. 3]. As a result, the effective segment boundaries do not coincide with the geometrical boundaries and the segment electrodes collect charge carriers from volumina of different sizes. This reflects in the occupancies of the segments when the detector is irradiated.

\subsection{Measured occupancies}

A $28 \mathrm{kBq}{ }^{228} \mathrm{Th}$ and a $40 \mathrm{kBq}{ }^{60} \mathrm{Co}$ source were used. The occupancies were measured for the $0.58 \mathrm{MeV}$ and $2.61 \mathrm{MeV}$ lines from ${ }^{208} \mathrm{Tl}$ and the $1.17 \mathrm{MeV}$ and $1.33 \mathrm{MeV}$ lines from ${ }^{60} \mathrm{Co}$. Only events, in which one segment registered the same energy, $E_{s}$, as the core within $50 \mathrm{keV}$, and the energy seen in any other segment was less than $150 \mathrm{keV}$ were used. As the separately measured background spectra did not show lines associated to ${ }^{60} \mathrm{Co}$, the background was not subtracted for the analysis of these lines. The background did show lines associated to ${ }^{208} \mathrm{Tl}$ decays. Therefore the background was subtracted for the analysis of the corresponding spectra. Figure 3(a) shows the spectrum measured in segment 3 for the irradiation from top and the corresponding normalized background spectrum. The background contribution for this relatively low-energy line is maximal at the bottom of the detector. The resulting segment-spectra were fitted using the log-likelihood method. The fit function was a combination of a Gauss function of width $\sigma$ and a sigmoid:

$f\left(E_{s}\right)=\frac{A}{\sqrt{2 \pi} \sigma} e^{-\frac{\left(E_{s}-E_{\gamma}\right)^{2}}{2 \sigma^{2}}}+B+\frac{C}{e^{\frac{2\left(E_{s}-E_{\gamma}\right)}{\sigma}}+1}$,

where $E_{\gamma}$ is the energy of the line under study and $A, B, C$ are the free parameters. The sigmoid represents the background shape. It drops for energies $E_{s}>E_{\gamma}$. The occupancy, $D_{i}$, of each segment $i$ was taken as the fitted number of events under the peak, $D_{i}=A$. binwidth $^{-1}$.

Figure 3(b) shows the result of the fit to the backgroundsubtracted spectrum around $0.58 \mathrm{MeV}$ for the irradiation with ${ }^{208} \mathrm{Tl}$ from the top. The contributions from the terms associated to the parameters $A, B$ and $C$ are indicated. The area in the peak associated to the parameter $A$ is well defined and what enters the analysis. Figure 4 shows the spectra of all 18 segments for the irradiation with ${ }^{60} \mathrm{Co}$ from the top. ${ }^{2}$ Also shown are the fit results.

Figure 5(a) shows the resulting occupancies for the middle layer. A clear structure is visible. It is almost mirrored with respect to the boundary between segments 13 and 6 . This agrees with the naive expectation, because the $\langle 110\rangle$ axis is almost aligned with this boundary.

Figure 5(b) shows the measured occupancies for the $2.61 \mathrm{MeV}$ line in the middle layer for the irradiation from the side. The situation is more complicated in this case and there is no naive expectation for the influence of the transverse anisotropy. The occupancies were extracted for all layers and all energies and for both irradiation from the top and the side.

\subsection{Expected occupancies}

Events for the experimental setup as described in Sect. 2 were simulated with the MAGE [16] package and trajectories were computed with the pulse shape simulation package [9]. The package provides calculations of the electrical and weighting fields and uses experimental input and model based calculations to provide the mobilities, and thus the velocities, of holes and electrons at any given point in the detector. The orientation of the $\langle 110\rangle$ axis, $\phi_{\langle 110\rangle}^{\text {sim }}$, was an input parameter to the simulation of the drift. The endpoints of the resulting trajectories were used to assign each energy deposit of an event to a segment. The energies were summed for each segment. The event selection was performed as for the measurements.

The resulting expected occupancies for $\phi_{\langle 110\rangle}^{\mathrm{sim}}=-20^{\circ}$ and $\phi_{\langle 110\rangle}^{\mathrm{sim}}=30^{\circ}$ are shown in Fig. 6 for the irradiation from the top and the side. The occupancies depicted in Fig. 6 were computed for the middle layer, for which an average $\rho_{\text {imp }}=0.45 \cdot 10^{10} \mathrm{~cm}^{-3}$ was assumed. For the irradiation from the top, the result for the $1.33 \mathrm{MeV}$ line of ${ }^{60} \mathrm{Co}$ is shown. For the irradiation from the side, the $2.61 \mathrm{MeV}$ line of ${ }^{208} \mathrm{Tl}$ was chosen.

For the irradiation from the top, the simulation of the middle layer predicts occupancy patterns which are almost identical for segments $15-14-13$ and $6-5-4$. This reflects the $180^{\circ}$ degeneracy of the axis orientation. The mirror symmetry observed in data with respect to the 13-6 boundary disappears, if the $\langle 110\rangle$ axis is not aligned with this boundary.

\footnotetext{
${ }^{2}$ Segment 9 in the top layer has a significantly larger resolution and its occupancy is only used in the analysis of data obtained by irradiation from the top.
} 
Fig. 3 (a) Measured signal and background spectrum in segment 3 around $0.58 \mathrm{MeV}$ and (b) the corresponding fit to the background-subtracted measured spectrum with the function given in Eq. (3) (a)

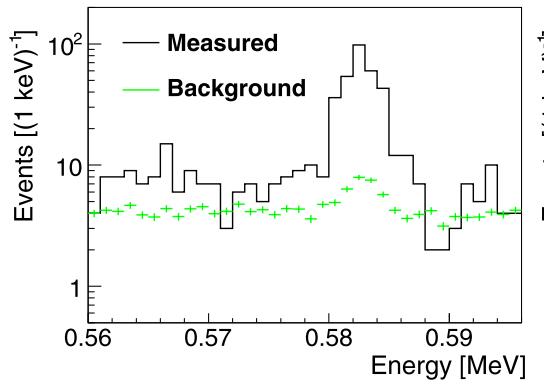

(b)

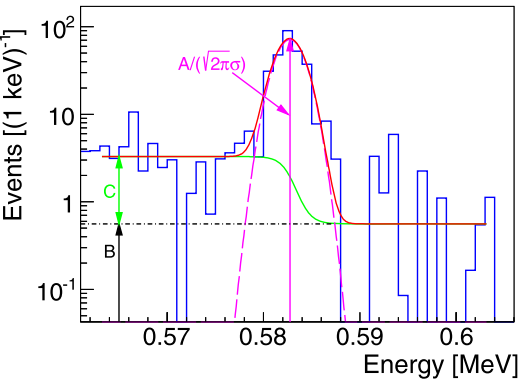

Fig. 4 Energy spectra at $1.33 \mathrm{MeV}$ as seen by the 18 segments for the irradiation with ${ }^{60} \mathrm{Co}$ from the top. Also shown are the results of the fits with the function given in Eq. (3)
Fig. 5 Measured occupancies (a) for the $1.33 \mathrm{MeV}$ line extracted for the middle layer for the irradiation from the top and (b) for the $2.61 \mathrm{MeV}$ line extracted for the middle layer for the irradiation from the side. The numbers denote the segment numbers
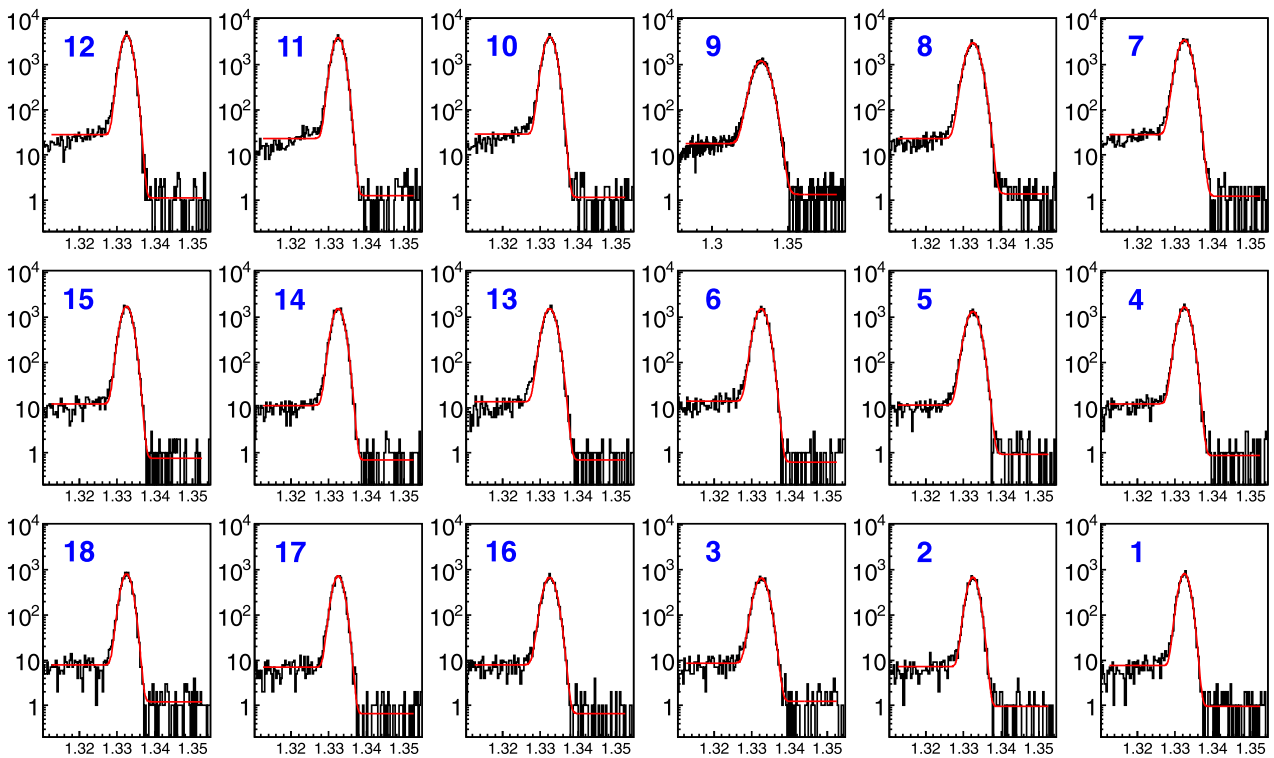

(a)

(b)

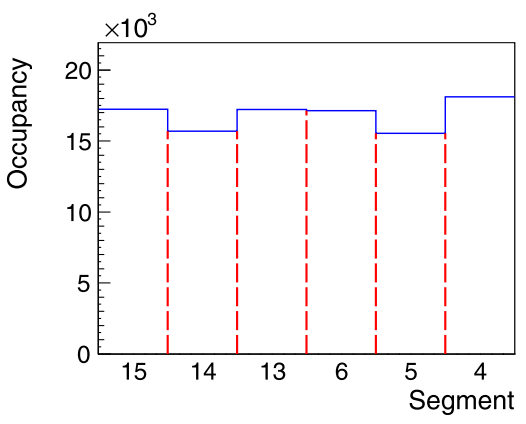

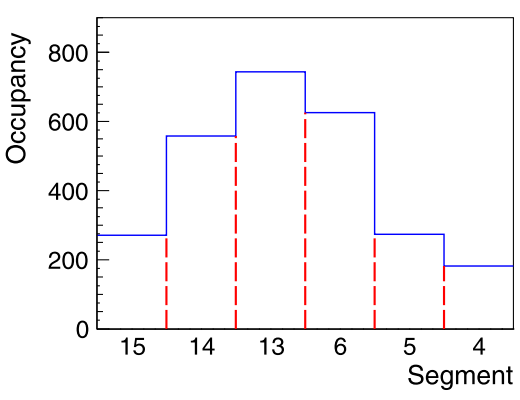

For the irradiation from the side, a clear difference between the predictions for $\phi_{\langle 110\rangle}^{\mathrm{sim}}=-20^{\circ}$ and $\phi_{\langle 110\rangle}^{\mathrm{sim}}=30^{\circ}$ is visible. There are no apparent symmetries, but the relative occupancies significantly change for varying $\phi_{\langle 110\rangle}$.

The amplitudes of the observed patterns depend on the amount of transverse anisotropy in the crystal. This is influenced by the hole mobility and by $\rho_{\text {imp. }}$. The dependence on $\rho_{\text {imp }}$ was investigated by comparing expectations for the nominal detector, i.e. the one supposed to be close to the real device with a $\rho_{\text {imp }}$ of $0.52 \cdot 10^{10} \mathrm{~cm}^{-3}, 0.45 \cdot 10^{10} \mathrm{~cm}^{-3}$ and
$0.38 \cdot 10^{10} \mathrm{~cm}^{-3}$, to predictions for a lower impurity test detector with a $\rho_{\text {imp }}$ of $0.29 \cdot 10^{10} \mathrm{~cm}^{-3}, 0.18 \cdot 10^{10} \mathrm{~cm}^{-3}$ and $0.06 \cdot 10^{10} \mathrm{~cm}^{-3}$ for the top, middle and bottom layers. Figures 7(a) and 7(b) show the expectations for $\phi_{\langle 110\rangle}^{\text {sim }}=0^{\circ}$.

The amplitude of a pattern can be defined as the depth of the first step of the pattern, $A_{i-j}=S_{i}-S_{j}$, with $S_{i}$ and $S_{j}$ being the predicted occupancies for the segments $i$ and $j$ located in the same layer. For the middle layer, $A_{15-14}=$ 0.0326 is predicted for the nominal detector and $A_{15-14}=$ 0.0336 for the test detector. This is a very small change of 
Fig. 6 Expected occupancy patterns in the middle layer for the irradiation from the top/side for $(\mathbf{a}) /(\mathbf{c}) \phi_{\langle 110\rangle}^{\mathrm{sim}}=-20^{\circ}$ and for (b) $/(\mathbf{d}) \phi_{\langle 110\rangle}^{\mathrm{sim}}=30^{\circ}$

(a)

(b)



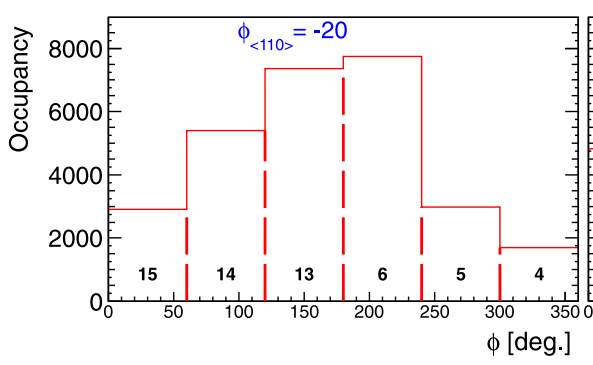

(c)

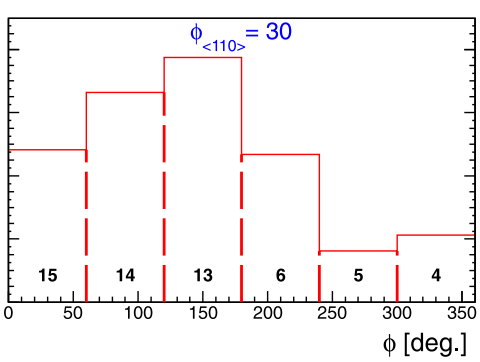

(d)

(a)

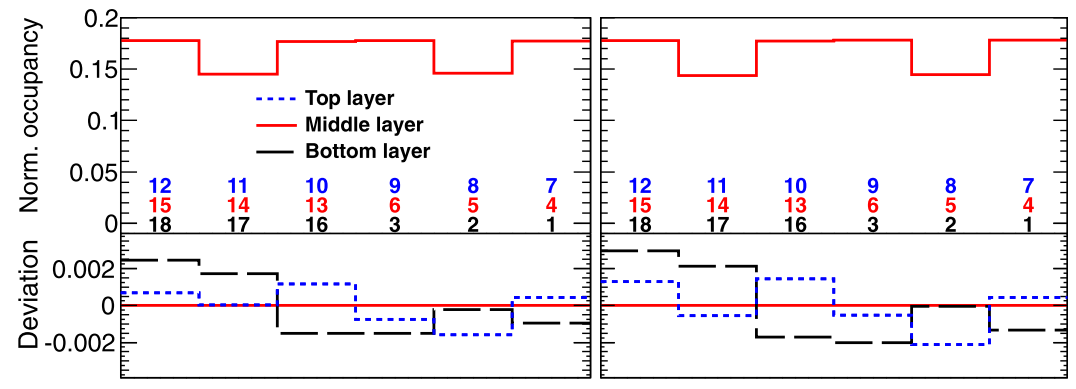

Fig. 7 Comparison of the normalized predicted occupancies for the $1.33 \mathrm{MeV}$ line and $\phi_{\langle 110\rangle}^{\text {sim }}=0^{\circ}$ for the middle, top and bottom layers of (a) the nominal crystal and (b) a test crystal with lower $\rho_{\text {imp }}$ degeneracy. The effect on a single occupancy is as much as 0.005 , which is a $3 \%$ effect on the occupancy, i.e. as much as $15 \%$ on the amplitude.

\subsection{Extraction of the axes orientation}

The occupancies were computed for $\phi_{\langle 110\rangle}^{\text {sim }}$ varying in $1^{\circ}$ steps. The resulting occupancies were analyzed by computing the test statistic $\epsilon$, defined as

$\epsilon=\sum_{i} \frac{\left(D_{i}-S_{i}\right)^{2}}{D_{i}^{2}}$,

where $D_{i}$ and $S_{i}$ denote the measured and simulated occupancies in segment $i$. The simulation was normalized to the total number of events in a given layer and the sum runs over all segments in this layer.

The resulting function $\epsilon\left(\phi_{\langle 110\rangle}^{\operatorname{sim}}\right)$ was fitted in a $20^{\circ}$ window with a second order polynomial. The $\phi_{\langle 110\rangle}^{\text {sim }}$ corresponding to the minimum of the polynomial, $\epsilon_{\min }$, was taken as the result of the procedure, $\phi^{\text {meas }}$. Figure 9 (a) shows $\epsilon\left(\phi_{\langle 110\rangle}^{\operatorname{sim}}\right)$ g $\phi=90^{\circ}$. The misalignments cause changes in pattern for the middle layer breaking the 15-14-13 6-5-4 
Fig. 8 Expected occupancies for $\phi_{\langle 110\rangle}^{\text {sim }}=0^{\circ}$ for the middle layer for (a) the $1.33 \mathrm{MeV}$ line and for (b) the $2.61 \mathrm{MeV}$ line for irradiation from the top. Also shown are the changes predicted for a $5 \mathrm{~mm}$ misalignment of the source along $45^{\circ}$ or $90^{\circ}$ (a)

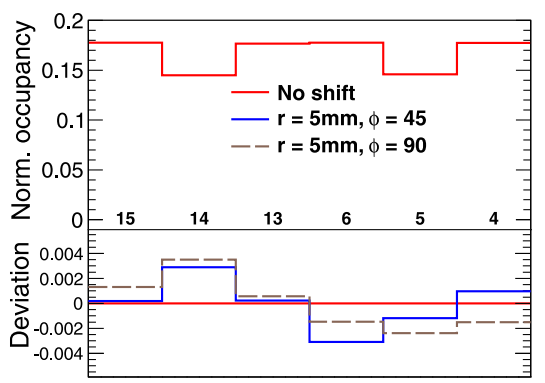

(a)



(b)

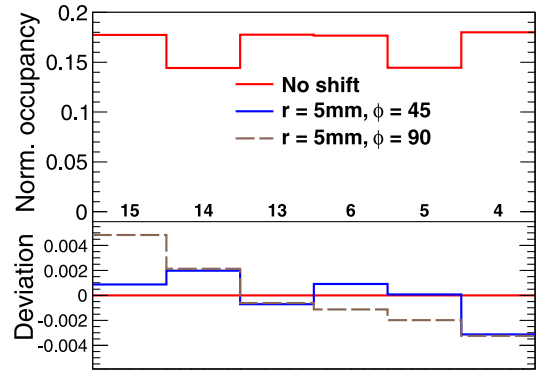

(b)

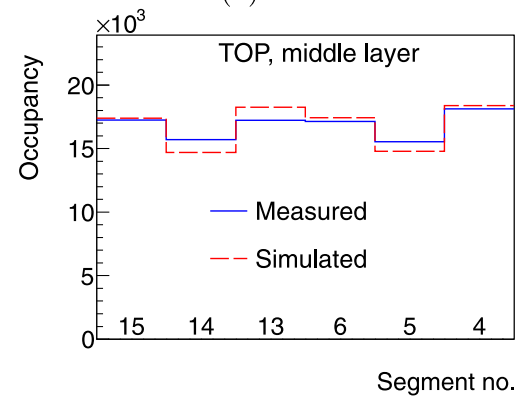

Fig. 9 (a) The dependence of $\epsilon$ on $\phi_{\langle 110\rangle}^{\text {sim }}$ for the $1.33 \mathrm{MeV}$ line in the middle layer for the irradiation from the top. (b) The corresponding measured and expected occupancies. The numbers denote the segment numbers. The simulation was normalized to the observed number of events in the middle layer plus the result of the polynomial fit for the case of the 1.33 MeV line in the middle layer for irradiation from the top. The shape of the pattern observed in the data is best reproduced by the prediction for $\phi_{\langle 110\rangle}^{\text {sim }} \approx 4^{\circ}$. Figure $9(\mathrm{~b})$ depicts the corresponding measured and predicted occupancies.

The comparison between the predicted and measured pattern in Fig. 9 shows that the shape of the pattern is very well reproduced. However, the amplitude of the predicted pattern is larger than the amplitude of the measured pattern. As was shown in Sect. 4.2, this cannot be explained by the choice of $\rho_{\text {imp }}$ in the simulation or by any misalignment of the source. As the hole mobility is not well known [8], its simulation could be the source of the discrepancy.

Figure 10 shows the situation for the $2.61 \mathrm{MeV}$ line in the middle layer for the irradiation from the side. The value of $\epsilon_{\text {min }}$ is significantly higher than for the top case depicted in Fig. 9, but the minimum is as distinct. The pattern observed in the data is well reproduced by the prediction for $\phi_{\langle 110\rangle} \approx$ $164^{\circ} \widehat{=}-16^{\circ}$.

\subsection{Systematic uncertainties}

The systematic uncertainties fall into two categories:

1. parameters used in the treatment of the data

2. imperfections of the simulation

The first category is dominated by uncertainties arising through the definition of $\epsilon$ and the fit to the resulting $\epsilon$ function. The test statistic $\epsilon$ deviates from a classical $\chi^{2}$ by putting extra emphasis on bins with lower occupancies. For the irradiation from the top, the differences in the results are small and the resulting systematic uncertainty is $1^{\circ}$. For the irradiation from the side, the differences in the results are significant. This is intended. However, a study using different test statistics yielded a systematic uncertainty of $4^{\circ}$. The effect of a variation of the width of the fit window for the
Fig. 10 (a) The dependence of $\epsilon$ on $\phi_{\langle 110\rangle}^{\text {sim }}$ for the $2.61 \mathrm{MeV}$ line in the middle layer for the irradiation from the side.

(b) The corresponding measured and expected occupancies. The numbers denote the segment numbers. The simulation was normalized to the observed number of events in the middle layer (a)

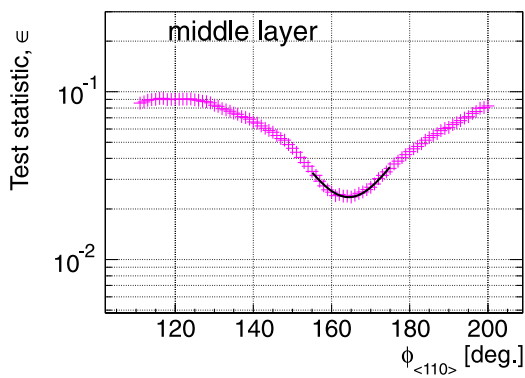

(b)

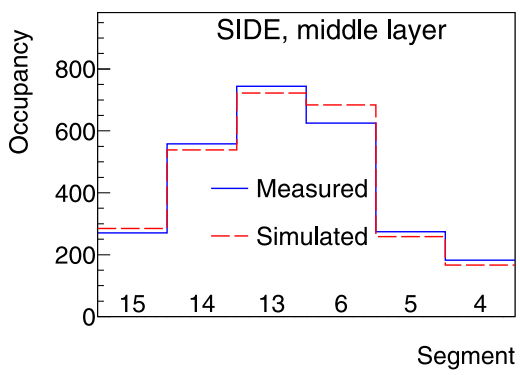


$\epsilon$-function was also studied. The window was widened to $30^{\circ}$ and narrowed to $10^{\circ}$ and the resulting uncertainty is $1^{\circ}$. Using other functions then a second order polynomial to fit the $\epsilon$-function yielded a systematic uncertainty of $2^{\circ}$.

The total systematic uncertainty connected with $\epsilon$ is evaluated by adding the individual uncertainties in quadrature. It is $2.5^{\circ}$ and $4.6^{\circ}$ for irradiation from the top and the side, respectively.

In the second category, the influence of the combination of hits before drifting the charge carriers was studied. Per default, hits within a distance of $1 \mathrm{~mm}$ were clustered. That was changed to $2 \mathrm{~mm}$ and the uncertainty due to this was found to be negligible. Any diffusion of the charge cloud is not simulated; the effect is expected to be small compared to the influences of charge clustering.

The main uncertainties are connected with the assumptions on the detector parameters and the source location.

As shown in Sect. 4.2, a mismatch of simulated and real $\rho_{\text {imp }}$ slightly changes the amplitude of the pattern, but not the shape. Therefore, the absolute values of $\epsilon$ will change, but the result will actually not. This was confirmed by simulation.

Also discussed in Sect. 4.2 was the influence of any misalignment of the source. The source position was controlled to about $5 \mathrm{~mm}$. Different layers are affected slightly differently due to the difference in angular coverage. The simulations show that the systematic uncertainty due to possible misalignments of up to $5 \mathrm{~mm}$ is $5^{\circ}$ for irradiation from the top.

The systematic uncertainty for the irradiation from the side directly reflects the uncertainty in the $\phi$-position of the source with respect to the detector. The detector was remounted for the side measurement and its relative rotation within the cryostat could, as mentioned before in Sect. 3, only be controlled to $3^{\circ}$. In addition, the position of the source relative to the cryostat could only be controlled to $5^{\circ}$. Added in quadrature, this results in a total systematic uncertainty of $6^{\circ}$.

For the analysis of lines which are also present in the background, possible changes in the background over time are another source of systematic uncertainty. Such changes can occur, if the cryostat or any other equipment in the lab is moved. Unfortunately, this was the case. The resulting patterns are very sensitive, especially for the irradiation from the side. The uncertainty was evaluated conservatively by performing the complete analysis for the ${ }^{208} \mathrm{Tl}$ lines with and without background subtraction. The result is a systematic uncertainty of $1^{\circ}$ and $7^{\circ}$ for the top and side cases, respectively.

In total, the systematic uncertainties for the irradiation from the top and the side were evaluated to be $6^{\circ}$ and $10^{\circ}$, respectively. They are dominated by the uncertainty in the source position and, for the irradiation from the side, possible changes in the background.

\subsection{Results}

The axes orientation was determined for all energies and all layers. The results, $\phi^{\text {meas }}$, are given in Table 1. Also given are weights, $w=\epsilon_{\min }^{-1}$, for all measurements. The statistical uncertainty of the fit to the $\epsilon$-function was always negligible compared to the systematic uncertainty.

For the irradiation from the top, the results for the ${ }^{208} \mathrm{Tl}$ lines show a spread which is slightly larger than expected from the evaluation of the systematic uncertainties. This could be due to the effect of statistical fluctuations in individual bins. An ensemble test was used to determine the statistical uncertainties. The test was performed by randomly varying the content of each bin according to its statistical uncertainty and extracting $\phi^{\text {meas }}$ for the modified occupancy pattern. The resulting $\phi^{\text {meas }}$ distribution was fitted with a Gaussian function and the result used to determine the statistical uncertainty. The largest uncertainties were observed for the $2.61 \mathrm{MeV}$ line for the irradiation from the top. They were $4^{\circ}$ for all layers.

The statistical and systematic uncertainties are different for different energies and layers. Therefore, weighted averages were computed:

$\phi_{\langle 110\rangle}^{\text {meas }}=\frac{\sum \phi_{\min }^{\text {meas }} \cdot w}{\sum w}$,

where the sums run over a combination of layers and line energies. The results for the ${ }^{60} \mathrm{Co}$ and ${ }^{208} \mathrm{Tl}$ lines in the three layers for irradiation from the top are:

$\phi_{\langle 110\rangle}^{\mathrm{Co} \text {,top }}=-1.8^{\circ} \pm 1^{\circ}($ stat $) \pm 6^{\circ}$ (syst),

$\phi_{\langle 110\rangle}^{\mathrm{Tl}, \text { top }}=-3.6^{\circ} \pm 4^{\circ}$ (stat) $\pm 6^{\circ}$ (syst).

Figure 11 shows the measured occupancies together with the best predictions for the middle and bottom layer for the ${ }^{60} \mathrm{Co}$ irradiation. The high statistics ${ }^{60} \mathrm{Co}$ distributions show that the predictions reproduce the shapes of all four measured patterns well, but that the predicted amplitudes are consistently too large. The measured amplitudes are about

Table 1 Extracted $\langle 110\rangle$-axis positions, $\phi^{\text {meas }}$, for different gamma lines and layers. Also listed are the weights, $w$, as defined in the text

\begin{tabular}{|c|c|c|c|c|c|c|}
\hline \multirow{2}{*}{$\begin{array}{l}E_{\text {line }} \\
{[\mathrm{MeV}]}\end{array}$} & \multicolumn{2}{|c|}{ Top layer } & \multicolumn{2}{|c|}{ Middle layer } & \multicolumn{2}{|c|}{ Bottom layer } \\
\hline & $\phi^{\text {meas }}$ & $w$ & $\phi^{\text {meas }}$ & $w$ & $\overline{\phi^{\mathrm{n}}}$ & $w$ \\
\hline
\end{tabular}

Irradiation from the top:

\begin{tabular}{lrrrrrr}
0.58 & $-11.1^{\circ}$ & 79 & $-8.6^{\circ}$ & 52 & $13.1^{\circ}$ & 52 \\
1.17 & $-7.7^{\circ}$ & 100 & $1.4^{\circ}$ & 115 & $-3.7^{\circ}$ & 167 \\
1.33 & $-7.1^{\circ}$ & 88 & $4.2^{\circ}$ & 99 & $1.1^{\circ}$ & 117 \\
2.61 & $0.3^{\circ}$ & 38 & $-6.2^{\circ}$ & 23 & $-6.3^{\circ}$ & 44 \\
\multicolumn{2}{l}{ Irradiation from the side: } & & & & \\
0.58 & $-21.9^{\circ}$ & 33 & $-14.2^{\circ}$ & 52 & $-23.4^{\circ}$ & 63 \\
2.61 & $-18.6^{\circ}$ & 19 & $-15.6^{\circ}$ & 43 & $-15.9^{\circ}$ & 24 \\
\hline
\end{tabular}


Fig. 11 Measured occupancies together with the best predictions for the irradiation with ${ }^{60} \mathrm{Co}$ from the top for

(a) $1.17 \mathrm{MeV}$, middle layer

(b) $1.33 \mathrm{MeV}$, middle layer

(c) $1.17 \mathrm{MeV}$, bottom layer

(d) $1.33 \mathrm{MeV}$, bottom layer (a)
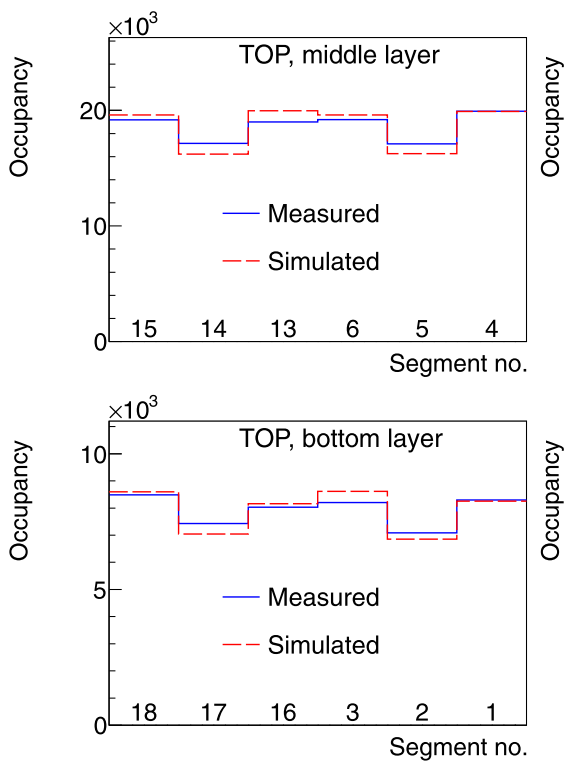

(c) (b)
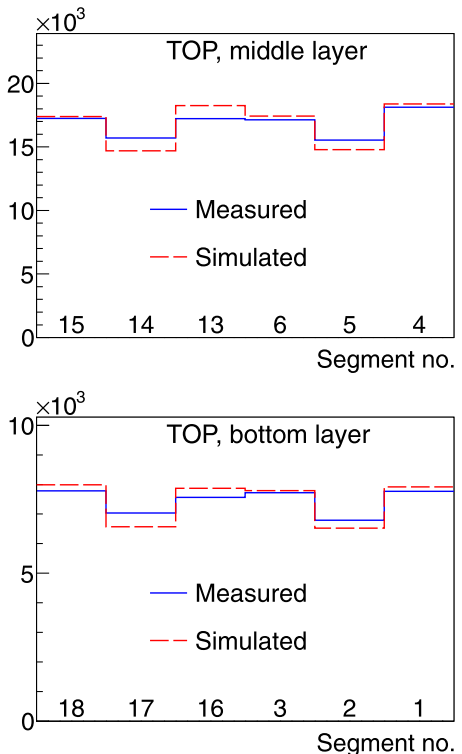

(d)
$40 \%$ smaller than the predicted ones. As discussed previously, this cannot be explained by an impurity level assumed to be too high in the simulation. The reproduction of the shapes of the measured distribution also indicates that the alignment of the source was well within the $5 \mathrm{~mm}$ uncertainty.

The simulation uses the hole mobilities as calculated by the pulse shape simulation package [9] using measured input parameters [8]. There is a non-negligible uncertainty in these calculations and the input parameters. The observation of less pronounced patterns than predicted could eventually be used to test the assumptions about hole mobilities. A simulation confirmed that the discrepancy between the predicted and observed amplitudes could be explained by an underestimate of the ratio of the mobilities along $\langle 111\rangle$ and $\langle 100\rangle$. The trajectories were recalculated for mobilities, for which the component along the $\langle 111\rangle$ axis was globally, i.e. independent of the electric field, increased by $10 \%$. This resulted in amplitudes about the size observed. However, as the calculation of mobilities along the $\langle 110\rangle$ axis and between the axes depend on several approximations, this will need further systematic studies.

Figure 12 shows the measured occupancies together with the best predictions for the middle and bottom layer for the ${ }^{208} \mathrm{Tl}$ irradiation from the top. The statistics is much lower than for the irradiation with ${ }^{60} \mathrm{Co}$, such that single bins can be affected by significant statistical fluctuations. The $2.61 \mathrm{MeV}$ line does not have a large probability to get fully absorbed in the volume of a single segment. The bottom layer has fewer events by design, because of the absorption in the crystal. It should be noted that the spread of the single measurements is still reasonable, even though single bins are fluctuating.

The individual values obtained for the irradiation from the side deviate consistently by more than $10^{\circ}$ from the expectation. The ensemble test for the $2.6 \mathrm{MeV}$ line, as discussed previously in this chapter, yielded the statistical uncertainty of $4^{\circ}$ and $6^{\circ}$ for the middle and bottom layers, respectively. Segment 9 was not used in the measurements using the top layer. Even though this segment was directly facing the source, the resulting measurements are still consistent with the results for the other layers. The top layer was, however, excluded from the averaging. The overall result is:

$\phi_{\langle 110\rangle}^{\mathrm{Tl} \text {, side }}=-17.9^{\circ} \pm 5^{\circ}$ (stat) $\pm 10^{\circ}$ (syst).

This result is barely consistent with the one obtained with the scan. Figure 13 depicts the measured occupancies together with the best predictions for the middle and bottom layer for the ${ }^{208} \mathrm{Tl}$ irradiation from the side. An irradiation directly from the side is a priori not favorable for this method. However, the patterns are reproduced quite well, indicating that probably the misalignment caused by the remounting of the detector and the changes in the background due to the movement of the equipment were larger than hoped for. The usage of this data for the analysis presented here was originally not planned. Therefore the systematic uncertainties relevant for this analysis were not really well controlled. With some care, they certainly could be reduced significantly.

The overall results confirm the ability to extract the axes orientation from the occupancy patterns in a $\phi$-segmented detector, even if the detector is not in a perfect condition. The precision depends on the care taken to avoid systematic 
Fig. 12 Measured occupancies together with the best predictions for the irradiation with ${ }^{208} \mathrm{Tl}$ from the top for

(a) $0.58 \mathrm{MeV}$, middle layer

(b) $2.61 \mathrm{MeV}$, middle layer

(c) $0.58 \mathrm{MeV}$, bottom layer

(d) $2.61 \mathrm{MeV}$, bottom layer

Fig. 13 Measured occupancies together with the best predictions for the irradiation with ${ }^{208} \mathrm{Tl}$ from the side for

(a) $0.58 \mathrm{MeV}$, middle layer

(b) $2.61 \mathrm{MeV}$, middle layer

(c) $0.58 \mathrm{MeV}$, bottom layer

(d) $2.61 \mathrm{MeV}$, bottom layer

(a)
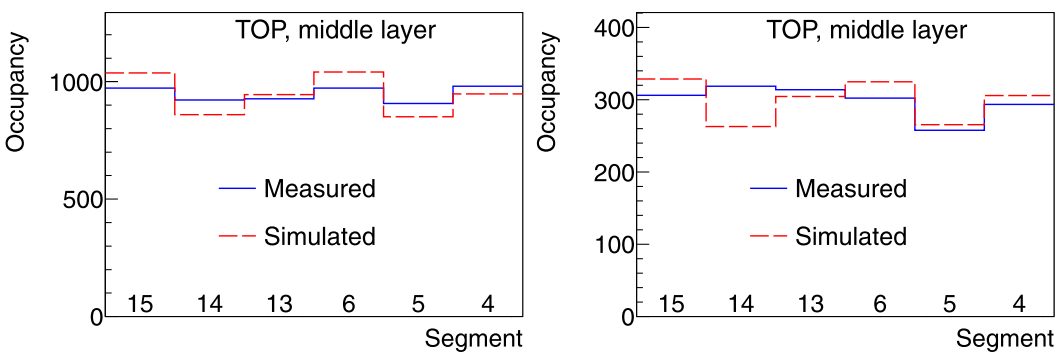

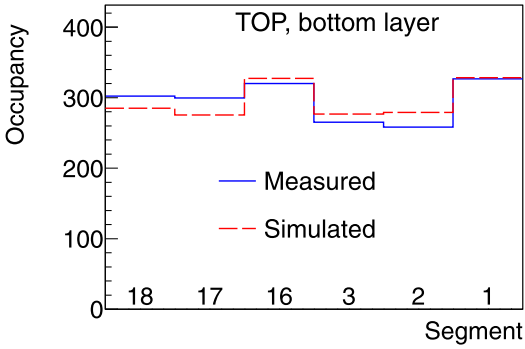

(c)

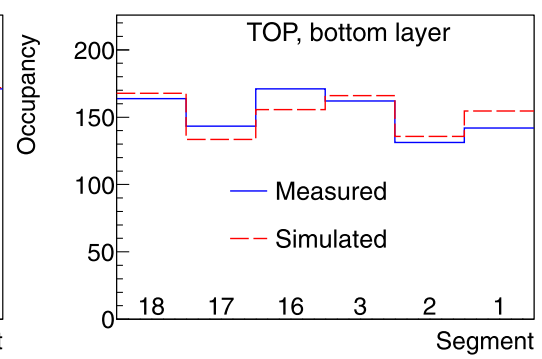

(d)

(b)
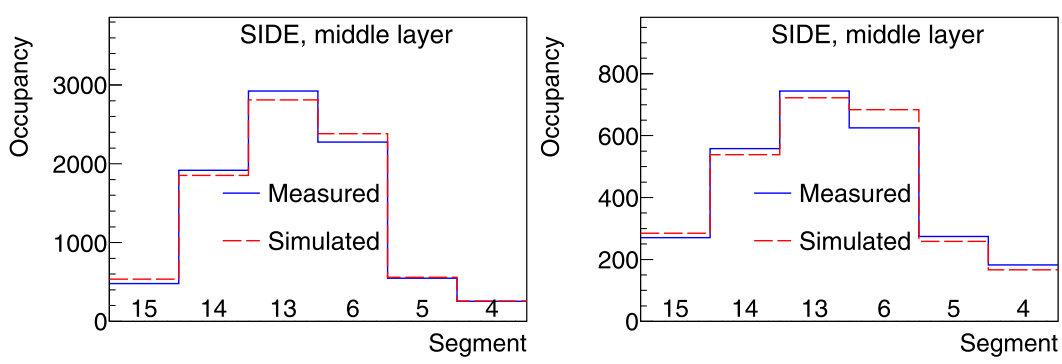

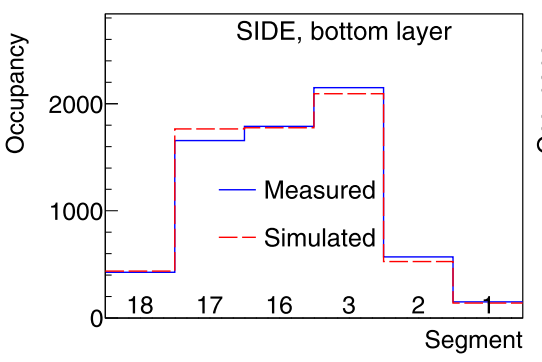

(c)

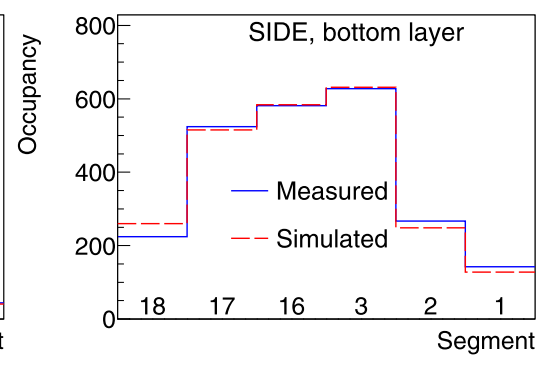

(d) uncertainties. The source position has to be well controlled under all circumstances. A symmetric configuration reduces the systematic uncertainties. The background has to be well known, if lines are used that also occur in the background. In general, a source should be chosen which has lines with a reasonably high probability of containment in a segment and high enough energy not to be disturbed too much by the surrounding material. The selection will depend on the setup and the detector. In the case considered here, the ${ }^{60}$ Co lines were found to be best suited.

\section{Conclusions}

A new method is presented to determine the axes orientation of $\phi$-segmented germanium detectors. The method relies on the ability to predict occupancy patterns caused by the transverse anisotropy of the drift of the relevant charge carriers. The axes orientation of a test detector was determined with a precision of about $6^{\circ}$. The precision was limited by the knowledge about the source locations. In general, a precision of $5^{\circ}$ should be attainable. This is compatible to the 
precision achieved with the well known scan method and is the precision needed for the usages of pulse shapes as currently envisioned. The new method can be used in any setup, also with multiple detectors, using any calibration source as long as the setup can be simulated with sufficient accuracy and background, if present, is properly taken into account. Therefore, it becomes unnecessary to perform time consuming scans to characterize detectors before they are integrated in a larger device. The method was developed and tested for true-coaxial detectors. However, it can be applied to any detector with an appropriate segmentation scheme. The modifications for a $\phi$-segmented closed-end cylindrical detector will be minimal.

The method has systematic uncertainties depending on the precision of the simulation of the setup and of the transverse anisotropy in the detector crystal. The dependence of the patterns on the transverse anisotropy provides the potential to determine charge carrier mobilities.

Acknowledgements We would like to thank the members of the MAGE Monte Carlo group for their kind support.
Open Access This article is distributed under the terms of the Creative Commons Attribution License which permits any use, distribution, and reproduction in any medium, provided the original author(s) and the source are credited.

\section{References}

1. G.F. Knoll, Radiation Detection and Measurement, 3rd edn. (Wiley, New York, 1999)

2. J. Eberth, J. Simpson, Prog. Part. Nucl. Phys. 60, 283 (2008)

3. I.-Y. Lee, J. Simpson, Nucl. Phys. News 20(4), 23 (2010)

4. I. Abt et al., arXiv:hep-ex/0404039

5. F. Recchia et al., Nucl. Instrum. Methods A 604, 555 (2009)

6. I. Abt et al., Nucl. Instrum. Methods A 583, 332 (2007)

7. I. Abt et al., Eur. Phys. J. A 36, 139 (2008)

8. B. Bruyneel et al., Nucl. Instrum. Methods A 569, 764 (2006)

9. I. Abt et al., Eur. Phys. J. C 68, 609 (2010)

10. I. Abt et al., Nucl. Instrum. Methods A 577, 574 (2007)

11. S.R. Elliott et al., Nucl. Instrum. Methods A 558, 504 (2006)

12. I. Abt et al., Eur. Phys. J. C 52, 19 (2007)

13. I. Abt et al., Nucl. Instrum. Methods A 570, 479 (2007)

14. I. Abt et al., J. Instrum. 4, 11008 (2009)

15. I. Abt et al., Eur. Phys. J. Appl. Phys. 56, 10104 (2011)

16. M. Boswell et al., IEEE Trans. Nucl. Sci. 58, 1212 (2011) 